\title{
Impact of community-based interventions for the prevention and control of malaria on intervention coverage and health outcomes for the prevention and control of malaria
}

Rehana A Salam¹, Jai K Das ${ }^{1}$, Zohra S Lassi ${ }^{1}$ and Zulfiqar A Bhutta ${ }^{2,3^{*}}$

\begin{abstract}
In this paper, we aim to evaluate the effectiveness of community-based interventions (CBIs) for the prevention and management of malaria. We conducted a systematic review and identified 42 studies for inclusion. Twenty-five of the included studies evaluated the impact of the community-based distribution of insecticide-treated nets (ITNs), indoor residual spraying (IRS), or impregnated bed sheets; 14 studies evaluated intermittent preventive therapy (IPT) delivered in community settings; two studies focused on community-based education for malaria prevention; and one study evaluated environmental management through drain cleaning.

Our analysis suggests that, overall, the community-based delivery of interventions to prevent and control malaria resulted in a significant increase in ITNs ownership (RR: $2.16,95 \% \mathrm{Cl}: 1.86,2.52)$ and usage (RR: $1.77,95 \%$ Cl: 1.48, 2.11). However, usage of ITNs was limited to two-thirds of the population who owned them. Community-based strategies also led to a significant decrease in parasitemia (RR: $0.56,95 \% \mathrm{Cl}: 0.42,0.74$ ), malaria prevalence (RR: 0.46 , 95\% Cl: $0.29,0.73$ ), malaria incidence (RR: $0.70,95 \%$ Cl: $0.54,0.90$ ), and anemia prevalence (RR: $0.79,95 \%$ Cl: 0.64 , 0.97). We found a non-significant impact on splenomegaly, birth outcomes (low birth weight, prematurity, stillbirth/miscarriage), anthropometric measures (stunting, wasting, and underweight), and mortality (all-cause and malaria-specific). The subgroup analysis suggested that community-based distribution of ITNs, impregnated bed sheets and IRS, and IPT are effective strategies. Qualitative synthesis suggests that high coverage could be achieved at a lower cost with the integration of CBls with existing antenatal care and immunization campaigns. Community-based delivery of interventions to prevent and control malaria are effective strategies to improve coverage and access and reduce malaria burden, however, efforts should also be concerted to prevent over diagnosis and drug resistance.
\end{abstract}

Keywords: Malaria, Community-based interventions, Malarial control, Malaria treatment

\section{Multilingual abstracts}

Please see Additional file 1 for translations of the abstract into the six official working languages of the United Nations.

\section{Introduction}

Malaria is a parasitic infection spread by the female Anopheles mosquito and is responsible for 660,000

\footnotetext{
* Correspondence: zulfiqar.bhutta@sickkids.ca

${ }^{2}$ Center of Excellence in Women \& Child Health, The Aga Khan University, Karachi, Pakistan

${ }^{3}$ Center for Global Child Health Hospital for Sick Children, Toronto, Canada Full list of author information is available at the end of the article
}

deaths globally and over 219 million cases of infection annually [1]. High-income countries (HICs) have been able to eliminate malaria, while many parts of low- and middle-income countries (LMICs) are still struggling to deal with malaria and vector control [1]. In 2012, out of the 104 malaria endemic countries, 79 countries are in the malaria control phase, ten are in the pre-elimination phase, ten are in the elimination phase, and five are focusing on the prevention of re-introduction [1]. A number of factors account for the existing malaria burden in developing countries including climate change, infrastructure, emerging drug and insecticide resistance, massive population and demographic shifts, and costs

\section{Biomed Central}

(c) 2014 Salam et al.; licensee BioMed Central Ltd. This is an Open Access article distributed under the terms of the Creative Commons Attribution License (http://creativecommons.org/licenses/by/2.0), which permits unrestricted use, distribution, and reproduction in any medium, provided the original work is properly credited. The Creative Commons Public Domain Dedication waiver (http://creativecommons.org/publicdomain/zero/1.0/) applies to the data made available in this article unless otherwise stated. 
of containment and therapy. In Sub-Saharan Africa, the wide-scale implementation of insecticide-treated bed nets (ITNs) has been one of the main strategies to reduce malaria morbidity and mortality [2].

There are existing interventions for malaria prevention, which include indoor residual spraying (IRS), ITNs, intermittent preventive therapy (IPT), presumptive treatment, and education. Previously, malarial chemoprophylaxis with chloroquine $(\mathrm{CQ})$ was generally recommended for pregnant women in malaria endemic regions, however, with the recent increase in Plasmodium falciparum resistance to CQ [3-5], the World Health Organization (WHO) recommends the use of sulfadoxine-pyrimethamine (SP) for the intermittent preventive treatment in pregnancy (IPTp). In this paper, we aim to evaluate the effectiveness of community-based interventions (CBIs) and their impact on the prevention and control of malaria.

\section{Methods}

We systematically reviewed literature published before May 2013 to identify studies evaluating the effectiveness of the community-based delivery of interventions for the prevention and control of malaria as outlined in our conceptual framework [6]. We included randomized controlled trials (RCTs), quasi-experimental trials, and before-andafter studies, in which the interventions for the prevention and control of malaria were delivered within community settings and the reported outcomes were relevant. A comprehensive search strategy was developed using appropriate keywords, medical subject headings $(\mathrm{MeSH})$, and free text terms. Searches were conducted in PubMed, Cochrane Libraries, Embase, and WHO Regional Databases. Studies that met the inclusion criteria were selected and double data abstracted on a standardized abstraction sheet. We excluded studies in which interventions were delivered in antenatal or immunization clinics, or if social marketing strategies in combination with facility-based interventions were evaluated. Studies were also excluded if the interventions were given to the displaced population or if the efficacy/effectiveness of a particular brand of bed nets, drugs, or diagnostic tools was evaluated. Studies reporting only entomological indices and parasite prevalence were also excluded. Quality assessment of the included RCTs was done using the Cochrane risk of bias assessment tool [7]. The outcomes of interest are outlined in Table 1. We conducted a meta-analysis for individual studies using the software Review Manager 5.1. Pooled statistics were reported as the relative risk (RR) for categorical variables and standard mean difference (SMD) for continuous variables between the experimental and control groups with 95\% confidence intervals (CIs). We also attempted to qualitatively synthesize the findings reported in the included studies for other pragmatic parameters identified in our conceptual framework including intervention coverage, challenges/barriers, enabling factors, aspects related to integrated delivery, monitoring and evaluations and equity. The detailed methodology is described in a separate paper [6].

\section{Review}

We identified 1,146 titles from the search conducted in all databases. After screening the titles and abstracts, 187 full texts were reviewed, of which 42 studies (17 RCTs, 10 quasi-experimental trials, 13 before-and-after studies, and two case control studies) were included in the review (see Figure 1). The characteristics of the included studies are summarized in Table 2. Of the 42 studies, four studies could not be included in the metaanalysis as these did not report poolable data [8-11], while for studies reporting multiple evaluations of a single intervention, we pooled the results from the last reported survey [12,13]. From the 17 RCTs included in this review, randomization was adequate in six studies, allocation was concealed in six studies, and adequate sequence generation was done in four studies. None of the studies blinded participants due to the nature of the interventions, while all studies provided insufficient information on selective reporting which limited us from making any judgment (see Table 3).

Twenty-five of the included studies evaluated the impact of the community-based distribution of ITNs or impregnated bed sheets, 14 studies evaluated IPT delivered in community settings, two studies focused on

Table 1 Outcomes analyzed

\begin{tabular}{ll}
\hline Outcomes & Outcomes analyzed \\
\hline Morbidity & Parasitemia \\
& Malaria incidence \\
& Malaria prevalence \\
& Splenomegaly \\
Anthropometry & Weight \\
& Height \\
Stunting \\
Wasting \\
Underweight \\
Prevalence of anemia \\
Birth Outcomes & Mean hemoglobin \\
Birth weight \\
Prematurity \\
Low birth weight (LBW) \\
Stillbirth/miscarriage \\
All-cause mortality \\
Malaria specific mortality \\
ITNs ownership \\
ITNs usage (sleeping under bed nets) \\
\end{tabular}




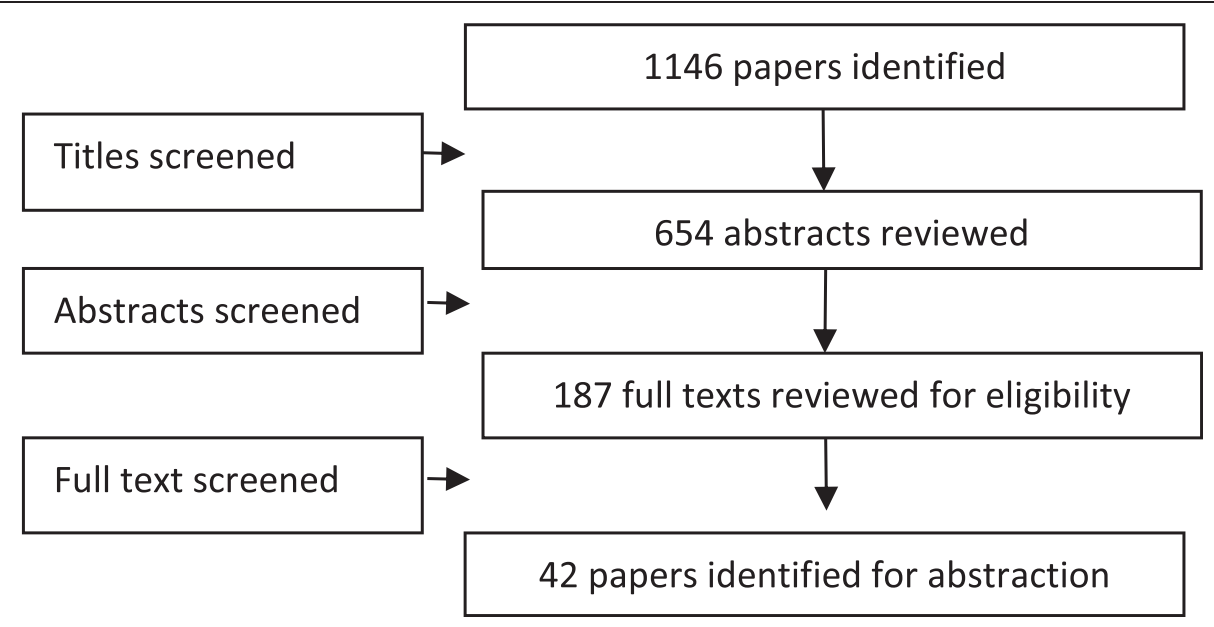

Figure 1 Search flow diagram.

community-based education for malaria prevention, and one study evaluated environmental management through drain cleaning. Community education on malaria preventive measures was one of the components of interventions in most of the studies. All the studies were conducted in African countries except for three studies, one each from China, India, and Thailand. Interventions were non-integrated in 23 [10,12-14,16-18,20-22,28-33,37,39-42, $45,47]$ of the studies, while in $17[8,9,19,23-27,34-36,43,44,46$, 48-50] studies intervention was integrated with routine community-based antenatal care (ANC), primary healthcare (PHC), child health days or measles, and polio campaigns. The primary comparison was between the communitybased delivery strategy versus routine or facility-based care. We also attempted to conduct a subgroup analysis to determine the relative effectiveness of integrated and nonintegrated delivery strategies, according to the type of intervention and whether the evidence was from RCT/ quasi-experimental studies or pre-post studies, where possible. The results are summarized in Tables 4 and 5 .

\section{Quantitative synthesis}

Table 4 depicts the impact of the overall communitybased delivery of interventions and the subgroup analysis according to the type of study and intervention. Overall, community-based delivery of interventions to prevent and control malaria resulted in a significantly higher ownership (RR: $2.16,95 \%$ CI: 1.86, 2.52) and usage (RR: 1.77, 95\% CI: $1.48,2.11$ ) of ITNs in the intervention group as compared to the control group (see Figures 2 and 3). Ownership was defined as households having at least one net at the time of the survey, while usage was defined as having slept under an ITN the previous night or having an ITN hanging over the bed. The usage rate of ITNs among people who owned an ITN was around 66\%. Community- based delivery strategy was also associated with significantly lower malaria incidence (RR: 0.70, 95\% CI: 0.54, 0.90 ), parasitemia (RR: $0.56,95 \%$ CI: $0.42,0.74$ ), and malaria prevalence (RR: $0.46,95 \%$ CI: $0.29,0.73$ ) in the intervention group (see Figures 4 and 5). Anemia prevalence also reduced significantly (RR: 0.79, 95\% CI: 0.64, 0.97) with sensitivity analysis after removing Eriksen 2010 (which reported concurrent interventions in both groups due to the introduction of a national campaign during the study period) (see Figure 6). We found non-significant impact on mean hemoglobin, splenomegaly, birth outcomes (low birth weight [LBW], prematurity, stillbirth/miscarriage), anthropometric measures (stunting, wasting, and underweight), and mortality (all-cause and malariaspecific). These findings are based on limited number of studies pooled with a high level of heterogeneity and hence should be interpreted with caution. The subgroup analysis for integrated and non-integrated delivery showed significant impacts on all outcome indicators except for malaria prevalence and splenomegaly which was nonsignificant for the integrated delivery subgroup, though this is based on a limited number of studies.

Table 5 summarizes the evidence from the subgroup analysis according to the type of intervention. The community-based distribution of ITNs, impregnated bed sheets, and IRS resulted in significantly higher ITNs ownership (RR: 2.28, 95\% CI: 1.95, 2.67), ITNs usage (RR: 2.49, 95\% CI: 1.90, 3.27), and mean hemoglobin levels (SMD: 9.00, 95\% CI: $8.80,9.20)$. It also led to a significant reduction in parasitemia (RR: $0.58,95 \%$ CI: $0.36,0.94$ ), malaria prevalence (RR: $0.42,95 \%$ CI: $0.25,0.70$ ), anemia prevalence (RR: $0.49,95 \%$ CI: $0.38,0.62$ ), and all-cause mortality (RR: 0.79, 95\% CI: 0.64, 0.96). The community-based delivery of IPT significantly reduced parasitemia (RR: 0.54, 95\% CI: $0.37,0.81$ ) and malaria-specific mortality 
Table 2 Characteristics of the included studies

\begin{tabular}{|c|c|c|c|c|c|}
\hline Study & Study design & Country & Intervention & Target population & Integrated/Non-integrated \\
\hline Abdulla 2001 [14] & Pre-post & Tanzania & $\begin{array}{l}\text { Social marketing about bed nets and ITNs } \\
\text { through public and private outlets and } \\
\text { door-to-door distribution }\end{array}$ & $\begin{array}{l}\text { Interventions for the general } \\
\text { population and outcomes } \\
\text { assessed in children under } \\
2 \text { years of age }\end{array}$ & Non-integrated \\
\hline Ahorlu 2009 [13] & Pre-post & Ghana & $\begin{array}{l}\text { Home delivered IPTc comprising of a single } \\
\text { dose of amodiaquine (AQ) + artesunate (AS) } \\
\text { over three days along with treatment of } \\
\text { febrile illness }\end{array}$ & Children 6-60 months of age & Non-integrated \\
\hline Ahorlu 2011 [12] & Pre-post & Ghana & $\begin{array}{l}\text { Home delivered IPTc comprising of a single } \\
\text { dose of } A Q+A S \text { over three days along with } \\
\text { treatment of febrile illness }\end{array}$ & Children 6-60 months of age & Non-integrated \\
\hline Ayi 2010 [15] & Quasi-experimental & Ghana & $\begin{array}{l}\text { School-based malaria education delivered } \\
\text { by teachers }\end{array}$ & School children in grades 3-5 & Non-integrated, school- based \\
\hline Bojang 2011 [16] & CRCT & Gambia & $\begin{array}{l}\text { IPTc delivered by village health workers (VHW) } \\
\text { comprising of a single dose of sulfadoxine } \\
\text { (SP) + three doses of AQ versus delivery through } \\
\text { reproductive and child health clinics }\end{array}$ & Children $<6$ years & Non-integrated \\
\hline Castro 2009 [17] & Quasi-experimental & Tanzania & $\begin{array}{l}\text { Environmental management through drain } \\
\text { cleaning versus use of larvicide versus } \\
\text { no intervention }\end{array}$ & General population & Non-integrated \\
\hline D’Alessandro 1995 [16] & CRCT & Gambia & Impregnated bed nets distribution & General population & $\begin{array}{l}\text { Integrated with PHC and delivered } \\
\text { through TBA }\end{array}$ \\
\hline D’Alessandro 1995 [18] & CRCT & Gambia & Impregnated bed nets distribution & Children $1-4$ years & $\begin{array}{l}\text { Integrated with PHC and delivered } \\
\text { through TBA }\end{array}$ \\
\hline D’Alessandro 1996 [19] & CRCT & Gambia & Impregnated bed nets distribution & Pregnant women & $\begin{array}{l}\text { Integrated with PHC and delivered } \\
\text { through TBA }\end{array}$ \\
\hline D’Alessandro 1997 [8] & Case control & Gambia & Impregnated bed nets distribution & Children 1-9 years of age & $\begin{array}{l}\text { Integrated with PHC and delivered } \\
\text { through TBA }\end{array}$ \\
\hline Dapeng 1996 [20] & Pre-post & China & Insecticide spraying + ITNs & General population & Non-integrated \\
\hline Delacollette 1996 [21] & Quasi-experimental & Zaire & $\begin{array}{l}\text { Educational messages + presumptive treatment } \\
\text { with chloroquine }(\mathrm{CQ}) \text { through CHWs versus } \\
\text { routine treatment in a health facility }\end{array}$ & General population & Non-integrated \\
\hline Eriksen 2010 [18] & CRCT & Tanzania & $\begin{array}{l}\text { Training of health workers and women leaders + } \\
\text { presumptive malaria treatment with single dose } \\
\text { SP versus routine facility care }\end{array}$ & Children $<5$ years & Non-integrated \\
\hline Ter Kuile 2003 [22] & CRCT & Kenya & ITNs distribution versus no distribution & Children $<36$ months & Non-integrated \\
\hline Gies 2008 [23] & Quasi-experimental & Burkina Faso & $\begin{array}{l}\text { Community based promotion + IPTp with SP } \\
\text { versus only IPTp with SP versus CQ }\end{array}$ & Pregnant women & Integrated with ANC \\
\hline Grabowski 2005 [24] & Pre-post & Ghana & ITNs distribution & Children $<5$ years & Integrated with measles campaign \\
\hline Grabowski 2005 [25] & Pre-post & Zambia & ITNs distribution & Children $<5$ years & Integrated with measles campaign \\
\hline
\end{tabular}


Table 2 Characteristics of the included studies (Continued)

\begin{tabular}{|c|c|c|c|c|c|}
\hline Greenwood 1989 [26] & $\mathrm{RCT}$ & Gambia & IPTp with Maloprim fortnightly delivered through TBA & Pregnant women & $\begin{array}{l}\text { Integrated with ANC delivered } \\
\text { through TBA }\end{array}$ \\
\hline Hawley 2003 [10] & $\mathrm{RCT}$ & Ghana & Community education + ITNs distribution & General population & Non-integrated \\
\hline Hightower 2010 [27] & Pre-post & Kenya & Free distribution & $\begin{array}{l}\text { Pregnant women and } \\
\text { children }<5 \text { years }\end{array}$ & Integrated with measles campaign \\
\hline Kidane and Morrow, 2000 [28] & $\mathrm{RCT}$ & Ethiopia & $\begin{array}{l}\text { Mothers trained to provide presumptive } \\
\text { treatment to children with CQ }\end{array}$ & Children $<5$ years & Non-integrated \\
\hline Kolaczinski 2010 [29] & Quasi-experimental & Uganda & ITNs distribution campaign & $\begin{array}{l}\text { Pregnant women and } \\
\text { children }<5 \text { years }\end{array}$ & Non-integrated \\
\hline Krezanoski 2010 [30] & cRCT & Madagascar & $\begin{array}{l}\text { Distribution of redeemable coupons } \\
\text { for ITNs }\end{array}$ & General population & Non-integrated \\
\hline Kuile 2003 [31] & $\mathrm{RCT}$ & Kenya & Distribution of ITNs versus no nets & $\begin{array}{l}\text { Intervention on general } \\
\text { population while outcomes } \\
\text { were assessed on children }\end{array}$ & Non-integrated \\
\hline Kweku 2009 [32] & cRCT & Ghana & $\begin{array}{l}\text { IPTC comprising of three doses of } \\
\mathrm{AQ}+\mathrm{SP} \text { delivered by community } \\
\text { volunteers versus delivery at outpatient } \\
\text { facilities or EPI clinics }\end{array}$ & Children $<5$ years & Non-integrated \\
\hline Macintyre 2003 [33] & $\mathrm{RCT}$ & Kenya & Use of impregnated bed sheets & General population & Non-integrated \\
\hline Mbonye 2008 [34] & Quasi-experimental & Uganda & $\begin{array}{l}\text { IPTp with two doses of SP delivered } \\
\text { through TBA shop vendors, community } \\
\text { reproductive health workers and APMs }\end{array}$ & Pregnant women & Integrated with ANC through TBAs \\
\hline Mbonye 2008 [35] & Quasi-experimental & Uganda & $\begin{array}{l}\text { IPTp with two doses of SP delivered } \\
\text { through TBA shop vendors, community } \\
\text { reproductive health workers and APMs }\end{array}$ & Pregnant women & Integrated with ANC through TBAs \\
\hline Msyamboza 2008 [36] & Quasi-experimental & Malawi & $\begin{array}{l}\text { IPTp comprising threw doses of SP } \\
\text { delivered through SP versus } \\
\text { facility-based care }\end{array}$ & Pregnant women & Integrated with ANC \\
\hline Noor 2007 [37] & Pre-post & Kenya & $\begin{array}{l}\text { ITNs delivered via the commercial sector } \\
\text { versus ANC clinics versus mass distribution }\end{array}$ & General population & Non-integrated \\
\hline Okabayashi 2006 [38] & Pre-post & Thailand & $\begin{array}{l}\text { School-based teacher training and manual } \\
\text { formulation for children, school lectures, } \\
\text { outdoor activities, and community } \\
\text { awareness about malaria prevention }\end{array}$ & $\begin{array}{l}\text { School children from } \\
\text { grades } 3-5\end{array}$ & School-based, non-integrated \\
\hline Okeibunor 2011 [39] & Quasi-experimental & Nigeria & $\begin{array}{l}\text { Distribution of IPTp (SP) + ITNs through } \\
\text { community volunteers }\end{array}$ & Pregnant women & Non-integrated \\
\hline Rhee 2005 [40] & Quasi-experimental & Mali & $\begin{array}{l}\text { Community education + net impregnation } \\
\text { services versus net impregnation alone }\end{array}$ & General population & Non-integrated \\
\hline Schellenberg 2001 [41] & Pre-post & Tanzania & $\begin{array}{l}\text { Social marketing of ITNs + insecticides for } \\
\text { ITNs through community shop keepers, } \\
\text { religious leaders, and health workers with } \\
\text { community sensitization }\end{array}$ & General population & Non-integrated \\
\hline
\end{tabular}


Table 2 Characteristics of the included studies (Continued)

\begin{tabular}{|c|c|c|c|c|c|}
\hline Sharma 2009 [42] & $\mathrm{RCT}$ & India & $\begin{array}{l}\text { Community group meeting for ITNs use } \\
\text { and mass awareness + olyset nets versus } \\
\text { untreated nets versus no nets }\end{array}$ & General population & Non-integrated \\
\hline Skarbinski 2007 [43] & Pre-post & Tanzania & $\begin{array}{l}\text { ITNs distribution with a child health } \\
\text { campaign including measles, vitamin } \\
\text { A, and deworming }\end{array}$ & Children $<5$ years & Integrated with child health campaign \\
\hline Staedke 2009 [44] & $\mathrm{RCT}$ & Uganda & $\begin{array}{l}\text { Home-based presumptive treatment with } \\
\text { artemether/lumefantrine versus clinic-based } \\
\text { routine care }\end{array}$ & Children $1-6$ years & Non-integrated \\
\hline Tagbor 2011 [45] & $\mathrm{RCT}$ & Ghana & $\begin{array}{l}\text { Presumptive malaria treatment by community } \\
\text { drug distributors (home based management) + } \\
\text { IPTC with AS and AQ }\end{array}$ & Children $<5$ years & Non-integrated \\
\hline Terlouw 2010 [46] & Pre-post & Togo & ITNs distribution with a child health campaign & Children $<5$ years & Integrated with child health campaign \\
\hline Thang 2009 [47] & cRCT & Vietnam & $\begin{array}{l}\text { Distribution of long-lasting insecticide-treated } \\
\text { hammocks }\end{array}$ & General population & Non-integrated \\
\hline Thwing 2008 [48] & Pre-post & Niger & ITNs distribution with polio immunization & Children $<5$ years & Integrated with polio immunization \\
\hline Wolkon 2010 [49] & Pre-post & Togo & $\begin{array}{l}\text { ITNs distribution with a deworming and } \\
\text { vaccine campaign during child health week }\end{array}$ & $\begin{array}{l}\text { Children + outcomes } \\
\text { in general population }\end{array}$ & Integrated with child health days \\
\hline
\end{tabular}


Table 3 Quality assessment of the included RCTs

\begin{tabular}{|c|c|c|c|c|c|c|}
\hline Study & Randomization & $\begin{array}{l}\text { Sequence } \\
\text { generation }\end{array}$ & $\begin{array}{l}\text { Allocation } \\
\text { concealment }\end{array}$ & $\begin{array}{l}\text { Blinding of } \\
\text { participants }\end{array}$ & $\begin{array}{l}\text { Blinding of } \\
\text { assessors }\end{array}$ & $\begin{array}{l}\text { Selective } \\
\text { reporting }\end{array}$ \\
\hline Bojang 2011 [16] & Done & Done & Not done & Not done & Not clear & No \\
\hline D'Alessandro 1995 [50] & Not clear & Not clear & Not done & Not done & Not done & No \\
\hline D’Alessandro 1995 [9] & Not clear & Not clear & Not done & Not done & Not done & No \\
\hline D’Allessandro 1996 [19] & Not clear & Not clear & Not done & Not done & Not done & No \\
\hline Eriksen 2010 [18] & Done & Done & Not done & Not done & Not clear & No \\
\hline Ter Kuile 2003 [31] & Done (Not clear) & Not clear & Not done & Not done & Not clear & No \\
\hline Greenwood 1989 [26] & Not clear & Not clear & Not done & Not done & Not clear & No \\
\hline Hawley 2003 [10] & Done (Not clear) & Not clear & Not done & Not done & Not clear & No \\
\hline Kidane 2000 [28] & Done (Not clear) & Not clear & Not done & Not done & Not clear & No \\
\hline Krezonoski 2010 [30] & Done & Not done & Not done & Not done & Not clear & No \\
\hline Kuile 2003 [22] & Done (Not clear) & Not clear & Not done & Not done & Not clear & No \\
\hline Kweku 2009 [32] & Done & Not done & Not done & Not done & Not clear & No \\
\hline Macintyre 2003 [33] & Done (Not clear) & Not clear & Not done & Not done & Not clear & No \\
\hline Sharma 2009 [42] & Done (Not clear) & Not clear & Not done & Not done & Not clear & No \\
\hline Staedke 2009 [44] & Done & Done & Not done & Not done & Not clear & No \\
\hline Tagbor 2011 [45] & Done (Not clear) & Not clear & Not done & Not done & Not clear & No \\
\hline Thang 2009 [47] & Done & Done & Not done & Not done & Not clear & No \\
\hline
\end{tabular}

(RR: 0.33, 95\% CI: 0.20, 0.55). Community education and cleanliness campaigns alone did not show any significant impact on the outcomes measured.

\section{Qualitative synthesis}

Interventions delivered in community setups reported great potential to improve coverage, access, and adherence to ITNs and IPT, as these were delivered via community volunteers who were easily accessible and trusted resource persons and who could make regular home visits and follow up with their patients [34]. Delivering intermittent preventive therapy during childhood (IPTc) through community health workers (CHWs) has shown several advantages as CHWs are community residents and can not only deliver effective and timely treatment, but also remind mothers/guardians if they forget to attend treatment. Thus, operationally, delivery using CHWs was less restrictive and more convenient for parents and guardians [16]. Furthermore, CHWs also contributed to improving recognition and referral of seriously ill patients, provided advice on hygiene and nutrition, and encouraged women to attend antenatal clinics and to immunize their children $[12,13,16]$. However, whether to give incentives to CHWs to encourage sustainability is still debated as some mass drug delivery systems have been successful without incentives whilst others have employed financial incentives of some kind [16]. Studies suggest that financial payment and a strengthened drug supply may contribute to program success, however, incentives must reach the CHWs in a timely and efficient manner to avoid demotivation
[16]. Besides incentivizing, CHWs require proper training, facilitation, and linkage with health units coupled with the provision of reference manual for easy and quick referencing to deliver timely treatment $[12,13,34]$.

The integration of CBIs for malaria with existing ANC and immunization campaigns is reportedly more feasible and acceptable, and has reported improved coverage of IPTp to pregnant women [36]. The integration of malaria control programs with such promotional campaigns has resulted in a major increase in treatment coverage and ITNs distribution at a very low cost $[23,24,36,43]$. Several features of ITNs distribution and mass measles vaccination campaigns favor the sustainability of an integrated approach. These include high coverage and low cost, as well as the fact that ITNs require replacing at the same intervals as measles vaccination campaigns take place $[24,27]$. However, effective integration requires careful planning to ensure that each component of the package is not jeopardized by the other [37]. Some of the other strategies shown to achieve high and equitable coverage include mass free distribution and social marketing [10,14,24,27,30,37,41].

Factors enabling the delivery of CBIs mainly involved community empowerment, intensive social mobilization, and education [24]. The implementation of environmental management activities at the community level requires empowering local residents, developing a sense of ownership, and improving environmental responsibility among the population [17]. Providing incentives, social marketing, and subsidization of the costs of ITNs have 
Table 4 Results for the overall and subgroup analysis according to the type of study and treatment

\begin{tabular}{|c|c|c|c|c|c|}
\hline \multirow[t]{2}{*}{ Outcomes } & \multicolumn{4}{|l|}{ Estimates $(95 \% \mathrm{CI})$} & \multirow[b]{2}{*}{$\begin{array}{l}\text { Community-based, } \\
\text { integrated delivery }\end{array}$} \\
\hline & Combined & RCTs & Pre-post studies & $\begin{array}{l}\text { Community-based, } \\
\text { non-integrated delivery }\end{array}$ & \\
\hline \multicolumn{6}{|l|}{ Coverage outcomes } \\
\hline ITNs ownership & $\begin{array}{l}\mathbf{2 . 1 6}[\mathbf{1 . 8 6}, \mathbf{2 . 5 2 ]}, 15 \\
\text { datasets } 14 \text { studies }\end{array}$ & $\begin{array}{l}0.97[0.94,1.00], 5 \\
\text { datasets } 4 \text { studies }\end{array}$ & $\begin{array}{l}3.71[2.62,5.27], 10 \\
\text { datasets } 10 \text { studies }\end{array}$ & $\begin{array}{l}1.24[1.11,1.39], 8 \\
\text { datasets } 7 \text { studies }\end{array}$ & $\begin{array}{l}5.05[2.59,9.86], 7 \\
\text { datasets } 7 \text { studies }\end{array}$ \\
\hline ITNs usage & $\begin{array}{l}\mathbf{1 . 7 7}[\mathbf{1 . 4 8 ,} \mathbf{2 . 1 1}], 16 \\
\text { datasets } 15 \text { studies }\end{array}$ & $\begin{array}{l}1.03[0.91,1.15], 9 \\
\text { datasets } 8 \text { studies }\end{array}$ & $\begin{array}{l}3.75[2.35,5.99], 7 \\
\text { datasets } 7 \text { studies }\end{array}$ & $\begin{array}{l}\mathbf{1 . 1 8}[1.03,1.34], 10 \\
\text { datasets } 9 \text { studies }\end{array}$ & $\begin{array}{l}6.97[3.10,15.69], 6 \\
\text { datasets } 6 \text { studies }\end{array}$ \\
\hline \multicolumn{6}{|l|}{ Morbidity outcomes } \\
\hline Parasitemia & $\begin{array}{l}\mathbf{0 . 5 6}[\mathbf{0 . 4 2 ,} \mathbf{0 . 7 4}], 11 \\
\text { datasets } 10 \text { studies }\end{array}$ & $\begin{array}{l}\mathbf{0 . 6 4}[\mathbf{0 . 4 8 ,} \mathbf{0 . 8 5}], 9 \\
\text { datasets } 8 \text { studies }\end{array}$ & $\begin{array}{l}0.15[0.01,2.56], 2 \\
\text { datasets } 2 \text { studies }\end{array}$ & $\begin{array}{l}\mathbf{0 . 3 9}[\mathbf{0 . 2 4}, \mathbf{0 . 6 4}], 5 \\
\text { datasets } 5 \text { studies }\end{array}$ & $\begin{array}{l}\mathbf{0 . 7 2}[0.53,0.99], 6 \\
\text { datasets } 5 \text { studies }\end{array}$ \\
\hline Malaria prevalence & $\begin{array}{l}\mathbf{0 . 4 6}[0.29, \mathbf{0 . 7 3}], 10 \\
\text { datasets } 9 \text { studies }\end{array}$ & $\begin{array}{l}0.52[0.32,0.85], 8 \\
\text { datasets } 7 \text { studies }\end{array}$ & $\begin{array}{l}0.29[0.05,1.78], 2 \\
\text { datasets } 2 \text { studies }\end{array}$ & $\begin{array}{l}\mathbf{0 . 4 2}[0.25,0.69], 9 \\
\text { datasets } 8 \text { studies }\end{array}$ & $\begin{array}{l}0.29[0.05,1.78], 1 \\
\text { dataset } 1 \text { study }\end{array}$ \\
\hline Malaria incidence & $\begin{array}{l}\mathbf{0 . 7 0}[\mathbf{0 . 5 4}, \mathbf{0 . 9 0}], 5 \\
\text { datasets } 5 \text { studies }\end{array}$ & $\begin{array}{l}\mathbf{0 . 7 0}[\mathbf{0 . 5 4}, \mathbf{0 . 9 0}], 5 \\
\text { datasets } 5 \text { studies }\end{array}$ & No studies & $\begin{array}{l}\mathbf{0 . 7 0}[\mathbf{0 . 5 4}, \mathbf{0 . 9 0}], 5 \\
\text { datasets } 5 \text { studies }\end{array}$ & No studies \\
\hline Splenomegaly & $\begin{array}{l}0.75[0.52,1.06], 4 \\
\text { datasets } 4 \text { studies }\end{array}$ & $\begin{array}{l}0.91[0.74,1.11], 3 \\
\text { datasets } 3 \text { studies }\end{array}$ & $\begin{array}{l}\mathbf{0 . 5 7}[0.49, \mathbf{0 . 6 5}], 1 \\
\text { dataset } 1 \text { study }\end{array}$ & $\begin{array}{l}\mathbf{0 . 5 7}[0.50,0.65], 2 \\
\text { datasets } 2 \text { studies }\end{array}$ & $\begin{array}{l}0.92[0.75,1.13], 2 \\
\text { datasets } 2 \text { studies }\end{array}$ \\
\hline \multicolumn{6}{|l|}{ Hematologic markers } \\
\hline Anemia prevalence & $\begin{array}{l}0.79[0.64,0.97], 10 \\
\text { datasets } 9 \text { studies }\end{array}$ & $\begin{array}{l}0.91[0.75,1.11], 9 \\
\text { datasets } 8 \text { studies }\end{array}$ & $\begin{array}{l}0.53[0.43,0.65], 2 \\
\text { datasets } 2 \text { studies }\end{array}$ & $\begin{array}{l}0.71[0.53,0.97], 6 \\
\text { datasets } 6 \text { studies }\end{array}$ & $\begin{array}{l}0.98[0.71,1.35], 5 \\
\text { datasets } 4 \text { studies }\end{array}$ \\
\hline Mean $\mathrm{Hb}$ & $\begin{array}{l}1.85[-0.85,4.55], 5 \\
\text { studies } 5 \text { studies }\end{array}$ & $\begin{array}{l}-0.03[-0.40,0.34], 4 \\
\text { datasets } 4 \text { studies }\end{array}$ & $\begin{array}{l}9.00[8.80,9.20], 1 \\
\text { dataset } 1 \text { study }\end{array}$ & $\begin{array}{l}2.22[-0.77,5.22], 4 \\
\text { datasets } 4 \text { studies }\end{array}$ & $\begin{array}{l}0.20[-1.80,2.20], 1 \\
\text { dataset } 1 \text { study }\end{array}$ \\
\hline \multicolumn{6}{|l|}{ Birth outcomes } \\
\hline Birth weight & $\begin{array}{l}22.68[-54.26,99.62], \\
3 \text { datasets } 3 \text { studies }\end{array}$ & $\begin{array}{l}22.68[-54.26,99.62], 3 \\
\text { datasets } 3 \text { studies }\end{array}$ & No studies & No studies & $\begin{array}{l}22.68[-54.26,99.62], \\
3 \text { datasets } 3 \text { studies }\end{array}$ \\
\hline LBW & $\begin{array}{l}0.95[0.63,1.44], 4 \\
\text { datasets } 3 \text { studies }\end{array}$ & $\begin{array}{l}0.95[0.63,1.44], 4 \\
\text { datasets } 3 \text { studies }\end{array}$ & No studies & No studies & $\begin{array}{l}0.95[0.63,1.44], 4 \\
\text { datasets } 3 \text { studies }\end{array}$ \\
\hline Prematurity & $\begin{array}{l}0.42[0.13,1.36], 1 \\
\text { dataset } 1 \text { study }\end{array}$ & $\begin{array}{l}0.42[0.13,1.36], 1 \\
\text { dataset } 1 \text { study }\end{array}$ & No studies & No studies & $\begin{array}{l}0.42[0.13,1.36], 1 \\
\text { dataset } 1 \text { study }\end{array}$ \\
\hline Stillbirth/miscarriage & $\begin{array}{l}1.23[0.90,1.69], 2 \\
\text { datasets } 1 \text { study }\end{array}$ & $\begin{array}{l}1.23[0.90,1.69], 2 \\
\text { datasets } 1 \text { study }\end{array}$ & No studies & No studies & $\begin{array}{l}1.23[0.90,1.69], 2 \\
\text { datasets } 1 \text { study }\end{array}$ \\
\hline \multicolumn{6}{|l|}{ Anthropometry outcomes } \\
\hline Weight & $\begin{array}{l}-0.02[-0.28,0.24], 2 \\
\text { datasets } 2 \text { studies }\end{array}$ & $\begin{array}{l}0.00[-0.28,0.28], 1 \\
\text { dataset } 1 \text { study }\end{array}$ & $\begin{array}{l}-0.10[-0.72,0.52], 1 \\
\text { dataset } 1 \text { study }\end{array}$ & $\begin{array}{l}-0.02[-0.28,0.24], 2 \\
\text { datasets } 2 \text { studies }\end{array}$ & No studies \\
\hline Stunting & $\begin{array}{l}1.11[0.86,1.42], 1 \\
\text { dataset } 1 \text { study }\end{array}$ & $\begin{array}{l}1.11[0.86,1.42], 1 \\
\text { dataset } 1 \text { study }\end{array}$ & No studies & $\begin{array}{l}1.11[0.86,1.42], 1 \\
\text { dataset } 1 \text { study }\end{array}$ & No studies \\
\hline Wasting & $\begin{array}{l}0.87[0.67,1.13], 1 \\
\text { dataset } 1 \text { study }\end{array}$ & $\begin{array}{l}0.87[0.67,1.13], 1 \\
\text { dataset } 1 \text { study }\end{array}$ & No studies & $\begin{array}{l}0.87[0.67,1.13], 1 \\
\text { dataset } 1 \text { study }\end{array}$ & No studies \\
\hline Underweight & $\begin{array}{l}0.94[0.78,1.14], 1 \\
\text { dataset } 1 \text { study }\end{array}$ & $\begin{array}{l}0.94[0.78,1.14], 1 \\
\text { dataset } 1 \text { study }\end{array}$ & No studies & $\begin{array}{l}0.94[0.78,1.14], 1 \\
\text { dataset } 1 \text { study }\end{array}$ & No studies \\
\hline \multicolumn{6}{|l|}{ Mortality } \\
\hline All-cause mortality & $\begin{array}{l}0.81[0.56,1.15], 3 \\
\text { datasets } 3 \text { studies }\end{array}$ & $\begin{array}{l}0.81[0.56,1.15], 3 \\
\text { datasets } 3 \text { studies }\end{array}$ & No studies & $\begin{array}{l}0.89[0.37,2.15], 1 \\
\text { dataset } 1 \text { study }\end{array}$ & $\begin{array}{l}\mathbf{0 . 7 9}[0.64, \mathbf{0 . 9 6}], 1 \\
\text { dataset } 1 \text { study }\end{array}$ \\
\hline Malaria specific mortality & $\begin{array}{l}0.54[0.21,1.40], 2 \\
\text { datasets } 2 \text { studies }\end{array}$ & $\begin{array}{l}0.54[0.21,1.40], 2 \\
\text { datasets } 2 \text { studies }\end{array}$ & No studies & $\begin{array}{l}\mathbf{0 . 3 3}[0.20, \mathbf{0 . 5 5}], 1 \\
\text { dataset } 1 \text { study }\end{array}$ & $\begin{array}{l}0.86[0.62,1.19], 1 \\
\text { dataset } 1 \text { study }\end{array}$ \\
\hline
\end{tabular}

also been reported as powerful tools, especially for programs seeking coverage for vulnerable groups such as children and pregnant women [30]. However, household ownership should be followed up to ensure usage. Studies have reported that barriers to ITNs use were not only the costs and access to ITNs, but also fear about insecticides and a lack of knowledge about malaria and ITNs
[27]. Other reasons for not using an ITN included discomfort, problems with hanging up the nets and lack of space, low awareness of its need, and seasonal variations in use [51]. Community education together with other interventions for malaria prevention can have a substantial impact on increasing the usage of ITNs [15]. Educational interventions based on lectures and theoretical case 
Table 5 Summary of evidence according to the type of intervention

\begin{tabular}{|c|c|c|c|}
\hline Outcomes & ITNs, bed sheets, and IRS & IPT & $\begin{array}{l}\text { Community education and } \\
\text { cleanliness campaigns }\end{array}$ \\
\hline ITNs ownership & $2.28[1.95,2.67]$ & & $0.99[0.76,1.28]$ \\
\hline ITNs usage & $2.49[1.90,3.27]$ & $1.07[0.59,1.94]$ & $1.02[0.96,1.09]$ \\
\hline Parasitemia & $0.58[0.36,0.94]$ & $0.54[0.37,0.81]$ & \\
\hline Malaria prevalence & $0.42[0.25,0.70]$ & $0.45[0.14,1.47]$ & $0.53[0.11,2.59]$ \\
\hline Malaria incidence & $0.74[0.53,1.04]$ & $0.50[0.22,1.14]$ & \\
\hline Splenomegaly & $0.72[0.44,1.17]$ & $0.82[0.52,1.32]$ & \\
\hline Anemia prevalence & $0.49[0.38,0.62]$ & $0.90[0.76,1.07]$ & \\
\hline Mean $\mathrm{Hb}$ & $9.00[8.80,9.20]$ & $-0.03[-0.40,0.34]$ & \\
\hline Weight & & $-0.02[-0.28,0.24]$ & \\
\hline Stunting & & $1.11[0.86,1.42]$ & \\
\hline Wasting & & $0.87[0.67,1.13]$ & \\
\hline Underweight & & $0.94[0.78,1.14]$ & \\
\hline All-cause mortality & $0.79[0.64,0.96]$ & $0.89[0.37,2.15]$ & \\
\hline Malaria specific mortality & $0.86[0.62,1.19]$ & $0.33[0.20,0.55]$ & \\
\hline
\end{tabular}

ITNs: Insecticide-treated nets, IRS: Indoor residual spraying, IPT: Intermittent preventive therapy.

*Estimates in bold suggests significant impact.

studies without any follow-up training have been shown to be less effective than multifaceted interventions involving other strategies [18]. School-based interventions involving school teachers delivering educational messages through activities such as role-playing, poetry recitals, slogan chanting, song composition, and dramatization have reportedly been acceptable and effective [15], however, these require a well-established school health system [15].

\section{Discussion}

Our review findings suggest that community-based delivery of interventions to prevent and control malaria is effective in improving ITNs ownership and usage, and reducing malaria incidence, parasitemia, malaria prevalence and anemia. However, this strategy did not have a statistically significant impact on birth outcomes, anthropometric measures, all-cause mortality, and malaria-

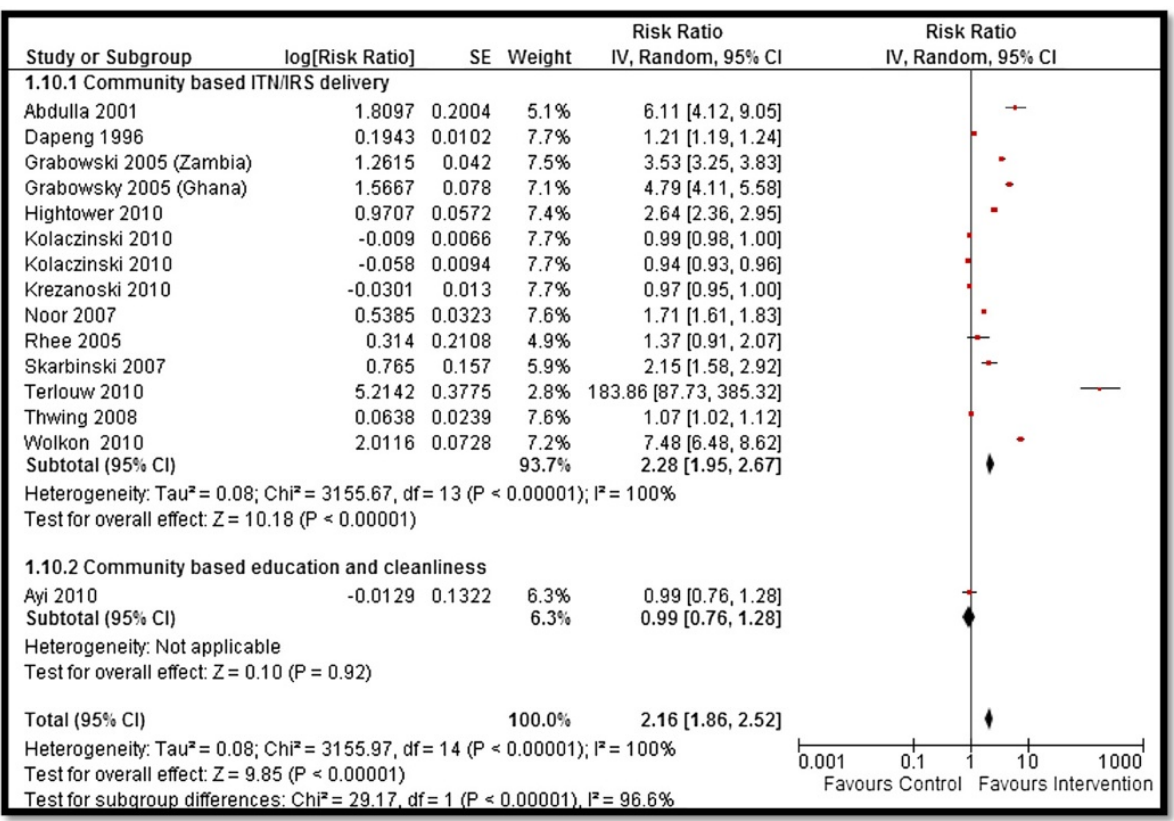

Figure 2 Forest plot for the impact of CBIs on ITNs ownership. 


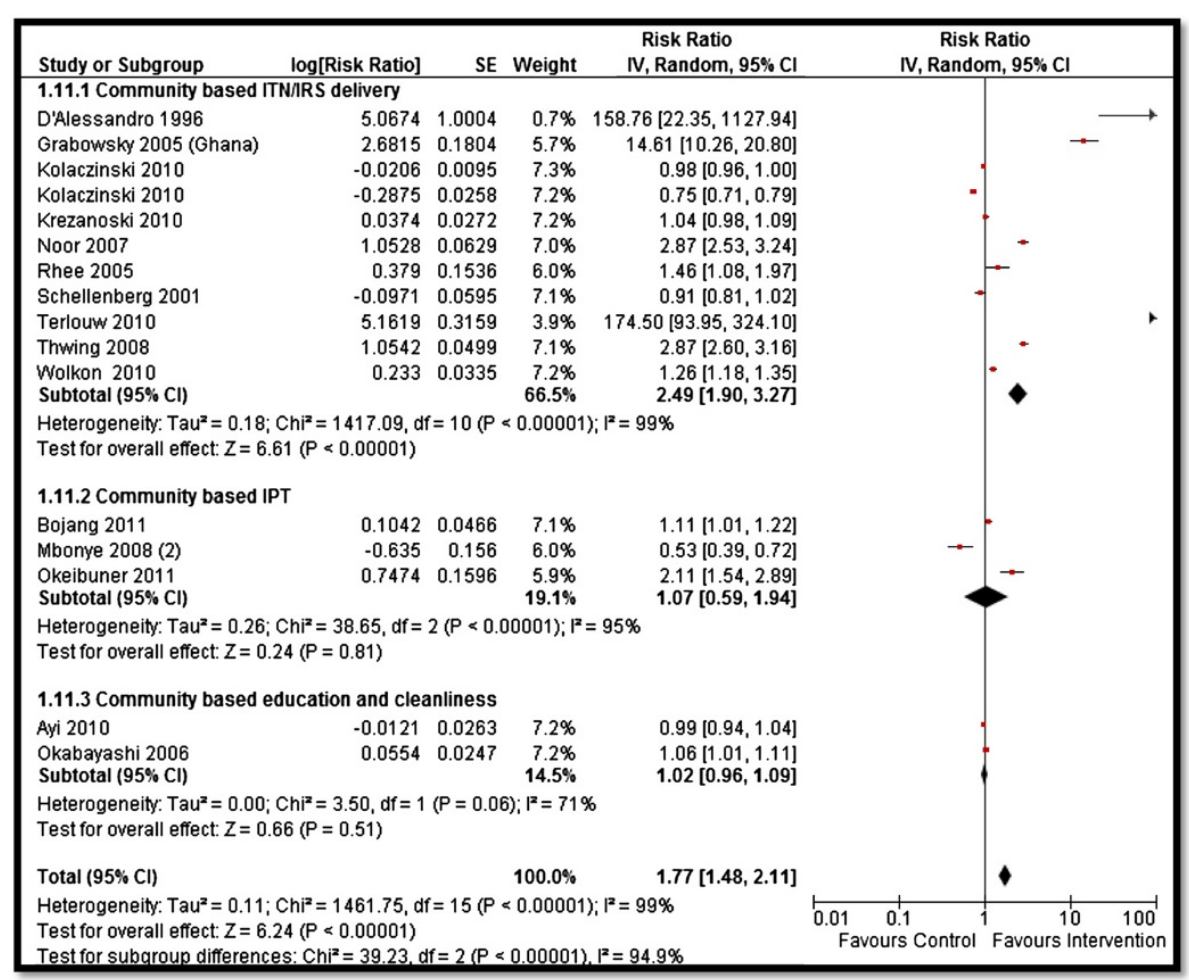

Figure 3 Forest plot for the impact of CBIs on ITNs usage.

specific mortality. These non-significant findings could be attributed to the concurrent national level malaria control measures being implemented in many of the African countries which could have led to more effective malaria control measures in both the intervention and control clusters since most of the studies included in our review are from Africa. Furthermore, the non-significant findings could also be attributable to the limited number of studies included for some of the outcomes (see Table 4). Our findings suggest a decrease in anemia prevalence,

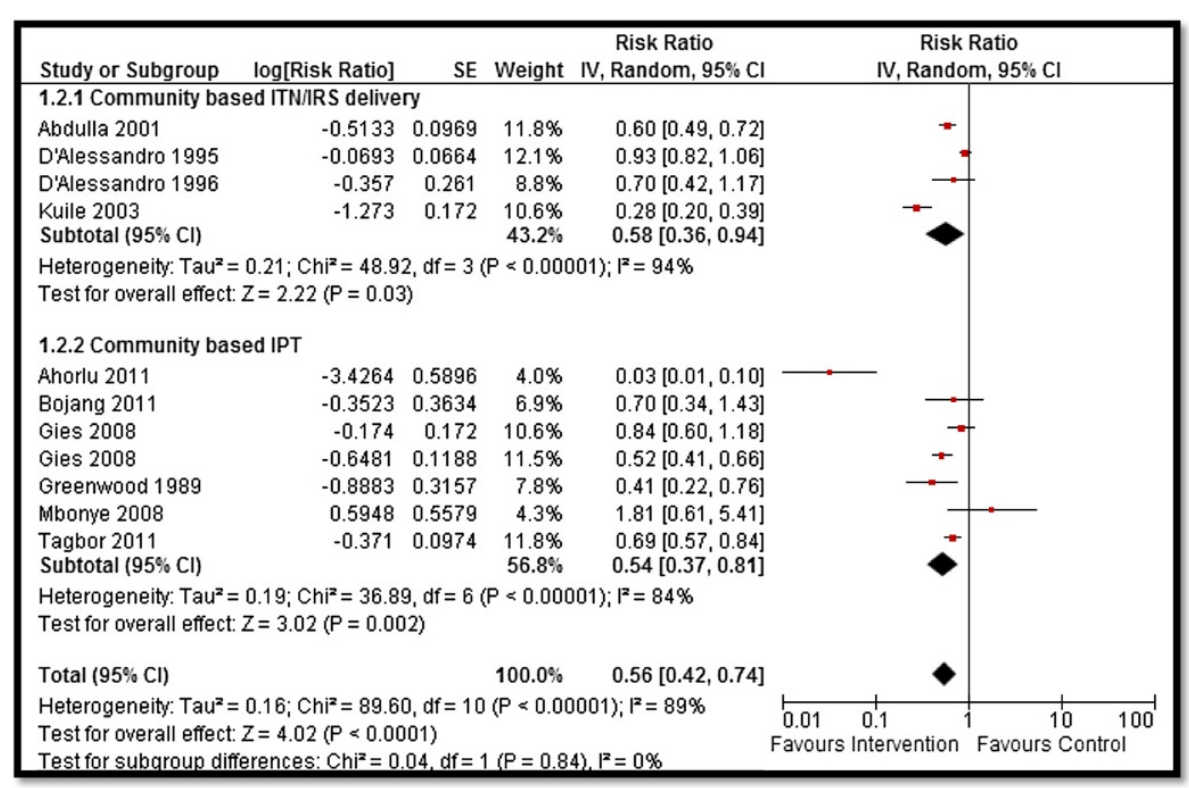

Figure 4 Forest plot for the impact of CBIs on parasitemia. 


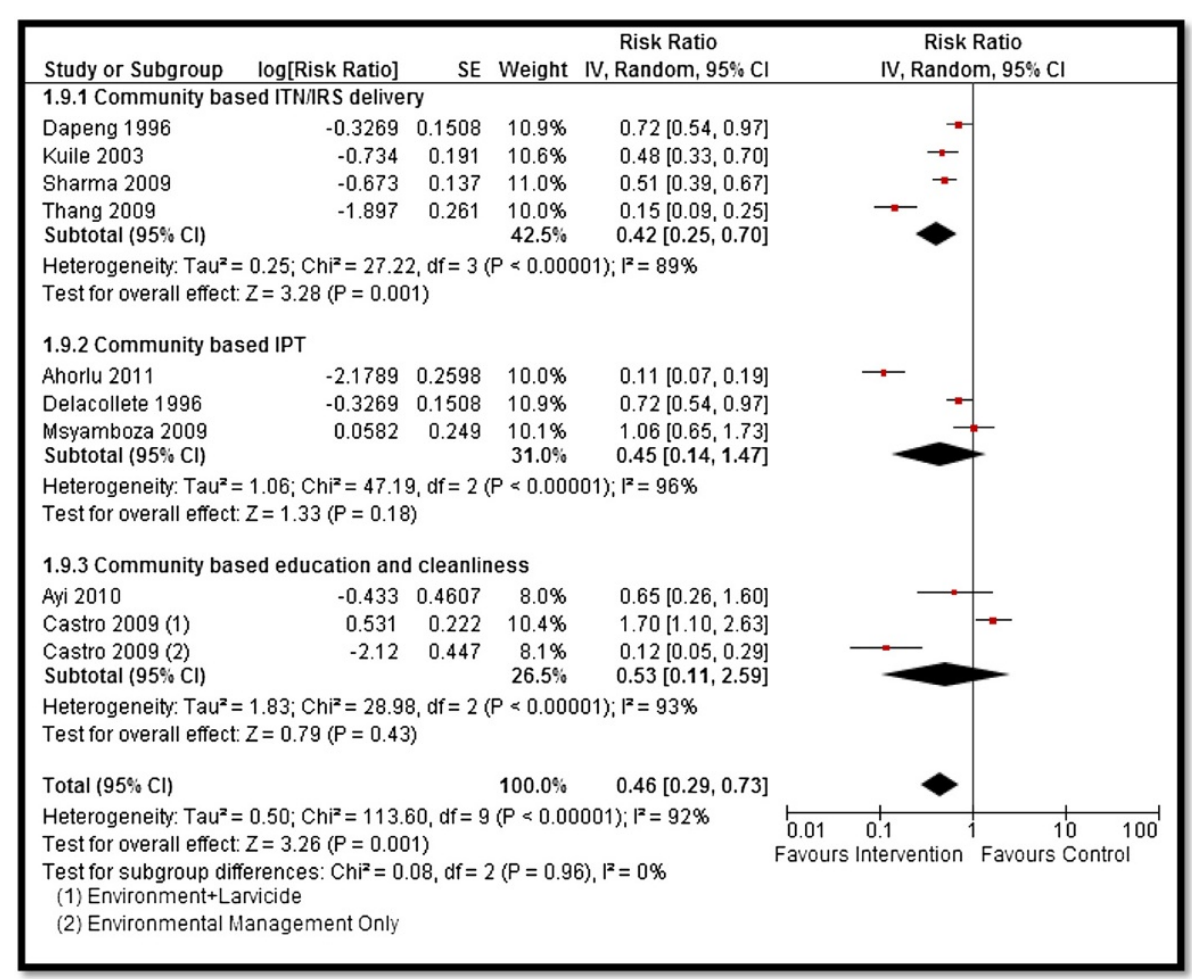

Figure 5 Forest plot for the impact of $\mathrm{CB}$ Is on malaria prevalence.

however, the mean hemoglobin remained non-significant. This could be due to various other causes of anemia coexisting with malaria in the study population (including infections, infestation, and malnutrition), the limited number of studies included, and a high level of heterogeneity in the pooled analysis. Such interactions and effect modifications should be considered when interpreting these findings.

Evidence from the subgroup analysis according to the type of interventions suggests that community-based distribution of ITNs, impregnated bed sheets, and IRS can effectively increase ITNs ownership, usage and mean hemoglobin levels, and effectively reduce parasitemia, malaria prevalence, anemia prevalence and all-cause mortality. The community-based delivery of IPT was found to be effective in reducing parasitemia and malaria-specific mortality, while community education and cleanliness campaigns alone did not show any improvement in the outcomes measured. The non-significant impact of the various types of interventions could also be attributable to the limited number of studies included for some of the outcomes in the subgroup analysis and a high level of heterogeneity. We did not find any conclusive evidence on the relative effectiveness of integrated and nonintegrated delivery strategies from our quantitative synthesis due to limited data in each subgroup. However, the qualitative synthesis of the data from the included studies suggests that high coverage could be achieved at a lower cost with the integration of CBIs with existing ANC and immunization campaigns. There is a need for large-scale effectiveness trials to explore the contextual parameters associated with scaling-up CBIs for malaria prevention and management.

Merely half of the countries with ongoing malaria transmission are on track to meet the World Health Assembly's (WHA's) target of 75\% reduction in malaria cases by 2015. The WHO recommends prompt and effective treatment with artemisinin-based combination therapies, use of ITNs by people at risk, and IRS with insecticide as the key interventions to control malaria. The past decade has witnessed some tremendous expansion in the implementation of malaria control programs with an increase in international disbursements from less than USD \$100 million in 2000 to USD \$1.71 billion in 2010, mainly targeting the African Region [1]. Treatment of malaria among children and pregnant women has also expanded coverage in many countries recently. However, millions of people still continue to lack access to preventive therapies, diagnostic testing, and quality-assured treatment with an emerging challenge being the resistance to artemisinins and mosquito resistance to insecticides. Some of the other reported factors affecting the delivery, access, and use of interventions to prevent malaria include unclear policy and guidance; general healthcare system issues, such as stock outs and user fees; health facility issues stemming 


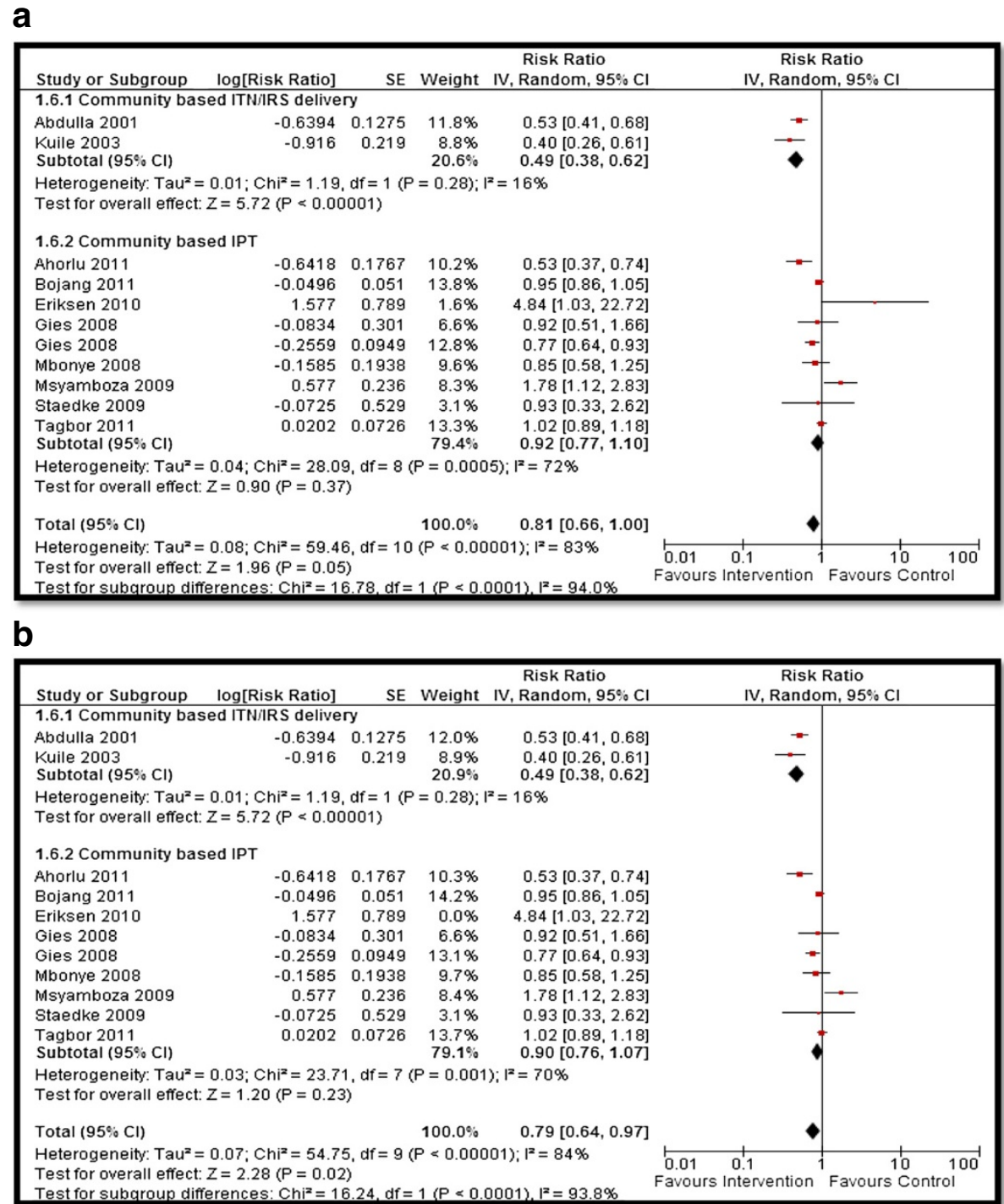

Figure 6 Forest plot for the impact of CBIs on anemia (a) with all studies included (b) after sensitivity analysis.

from poor organization and leading to poor quality of care; and poor healthcare provider performance. Key determinants of coverage include education, knowledge about malaria, socioeconomic determinants, and employment status [52-55].

With the recent increase in attention geared towards community-based delivery and its ever-expanding repertoire of services, systems must be identified to ensure training, support, and incentives for CBIs. Lack of supplies including ITNs and antimalarials have also been reported as a barrier to program success, and hence routine supply of these commodities needs to be guaranteed [16]. Another major barrier to sustainability of such programs is the issue of understaffing at health units [34]. There is also a need to address the challenges of fast growing cities and enhance the health conditions of its inhabitants [17]. Scaling-up environmental activities will require resources for initial massive cleaning and structural repairs that is not possible without donor support [17]. Community involvement remains an essential component of malaria control measures as these interventions require implementation at the household level and the disease is more prevalent in settings with limited access to health facilities. Building community ownership for creating demand for ITNs and increasing trust on CHWs is pivotal for any community-based program to be successful and improve health behaviors.

Interventions such as the distribution of ITNs, net impregnation, IPTp, and IPTc have the potential to be integrated with existing programs such as ANC, 
immunizations, deworming campaigns and child health days, and could provide useful models for evaluation. The WHO recommends seasonal malaria chemoprophylaxis to be delivered in integration with existing community-based programs, however, a single deployment strategy has not yet been devised [1]. Community case management $(\mathrm{CCM})$ that integrates the management of childhood diarrhea, pneumonia, and malaria is one of the strategies that has received government support and has the potential to improve access. This program utilizes existing $\mathrm{CHWs}$ to treat children during home visits and has led to an improvement in ITNs usage and timely malaria treatment for children [56-58]. Similarly, a sharp increase in the number of distributed ITNs have been observed during child health days $[59,60]$. Many countries in Africa have successfully implemented various integrated models of delivery, however, these are yet to be formally evaluated for effectiveness. A recent analysis carried out in Malawi, Rwanda, Kenya, and Senegal to determine the cost of providing integrated CCM concluded that this is associated with lower costs provided it is used by sufficient numbers of patients to justify the costs of training, equipping, managing, and supervising the CHWs who provide the services $[61,62]$. However, simultaneous efforts should also be concerted to prevent over diagnosis and drug resistance.

\section{Conclusion}

Collaborative partnerships between governments and donors for the establishment of healthier environments for malaria prevention could play a crucial role in building an ideal platform for malaria specific interventions. Such programs could be articulated in collaborative partnerships between the government and various institutions such as engineering, waste management, education, and public health. We conclude that community-based strategy to deliver malaria specific interventions including ITNs, IRS and IPT, in combination with community education and sanitation, can be effective in reducing the overall burden of malaria morbidity and mortality, especially in malaria endemic areas.

\section{Additional file}

Additional file 1: Multilingual abstracts in the six official working languages of the United Nations.

\section{Abbreviations}

ANC: Antenatal care; CBI: Community based intervention; Cl: Confidence interval; CQ: Chloroquine; Hb: Hemoglobin; CRCT: Cluster randomized controlled trial; HIC: High-income country; IDoP: Infectious diseases of poverty; IPTc: Intermittent preventive therapy during childhood; IPTp: Intermittent preventive therapy during pregnancy; IRS: Indoor residual spraying; ITN: Insecticide treated net; LMIC: Low- middle- income country; NTD: Neglected tropical disease; PHC: Primary health care; RBM: Roll back malaria; RCT: Randomized controlled trial; RR: Relative risk; SMD: Standard mean difference; SP: Sulfadoxine-pyrimethamine; VHW: Village health worker; WHO: World Health Organization.

\section{Competing interests}

The authors declare that they have no financial or non-financial competing interests.

\section{Authors' contributions}

ZAB was responsible for designing and coordinating the review. ZSL and RAS were responsible for the data collection, screening of the search results, screening of the retrieved papers against the inclusion criteria, appraising the quality of papers, and abstracting the data. RAS, JKD, and ZSL were responsible for data interpretation and writing the review. ZAB critically reviewed and modified the manuscript. All authors read and approved the final manuscript.

\section{Acknowledgements}

The collection of scoping reviews in this special issue of Infectious Diseases of Poverty was commissioned by the UNICEF/UNDP/World Bank/WHO Special Programme for Research and Training in Tropical Diseases (TDR) in the context of a Contribution Agreement with the European Union for "Promoting research for improved community access to health interventions in Africa".

\section{Author details}

'Division of Women and Child Health, The Aga Khan University, Karachi, Pakistan. ${ }^{2}$ Center of Excellence in Women \& Child Health, The Aga Khan University, Karachi, Pakistan. ${ }^{3}$ Center for Global Child Health Hospital for Sick Children, Toronto, Canada.

Received: 5 January 2014 Accepted: 22 July 2014

Published: 1 August 2014

\section{References}

1. WHO: WHO Global Malaria Programme: World Malaria Report 2011; 2012. Available at http://www.who.int/malaria/publications/world_malaria_report_ 2012/en/.

2. WHO: The Africa Malaria Report Malaria Control Unit, UNICEF. Geneva, Switzerland: World Health Organization; 2003.

3. Brabin BJGM, Alpers M, Brabin L, Eggelte T, Van der Kaay HJ: Failure of chloroquine prophylaxis for falciparum malaria in pregnant women in Madang, Papua New Guinea. Ann Trop Med Parasitol 1990, 84:1-9.

4. Sirima SB, Sawadogo R, Moran A, Konate A, Diarra A, Yameogo M, Parise ME, Newman RD: Failure of a chloroquine chemoprophylaxis program to adequately prevent malaria during pregnancy in Koupe'la District, Burkina Faso. Clin Infect Dis 2003, 36:1374-1382.

5. Heymann DL, Steketee RW, Wirima JJ, McFarland DA, Khoromana CO, Campbell C: Antenatal chloroquine chemoprophylaxis in Malawi: chloroquine resistance, compliance, protective efficacy and cost. Trans R Soc Trop Med Hyg 1990, 84:496-498.

6. Lassi SA, Salam RA, Das JK, Bhutta ZA: Paper 2: Conceptual Framework and Assessment Methodology for the Systematic Review on Community Based Interventions for the Prevention and Control of IDoP.

7. Higgins JPT, Green S: Cochrane Handbook for Systematic Reviews of Interventions Version 5.1.0. [updated March 201 1]. The Cochrane Collaboration. 2011. Available from [www.cochrane-handbook.org]

8. D'Alessandro U, Olaleye B, Langerock P, Bennett S, Cham K, Cham B, Greenwood BM: The Gambian national impregnated bed net programme: evaluation of effectiveness by means of case-control studies. Trans R Soc Trop Med Hyg 1997, 91(6):638-642.

9. D'Alessandro U, Olaleye BO, McGuire W, Thomson MC, Langerock P, Bennett S, Greenwood BM: A comparison of the efficacy of insecticide-treated and untreated bed nets in preventing malaria in Gambian children. Trans $R$ Soc Trop Med Hyg 1995, 89(6):596-598.

10. Hawley WA, Phillips-Howard PA, ter Kuile FO, Terlouw DJ, Vulule JM, Ombok M, Nahlen BL, Gimnig JE, Kariuki SK, Kolczak MS: Community-wide effects of permethrin-treated bed nets on child mortality and malaria morbidity in western Kenya. Am J Trop Med Hyg 2003, 68(4):121-127.

11. Ter Kuile FO, Terlouw DJ, Phillips-Howard PA, Hawley WA, Friedman JF, Kolczak MS, Kariuki SK, Shi YP, Kwena AM, Vulule JM: Impact of permethrin- 
treated bed nets on malaria and all-cause morbidity in young children in an area of intense perennial malaria transmission in western Kenya: cross-sectional survey. Am J Trop Med Hyg 2003, 68(4 suppl):100-107.

12. Ahorlu CK, Koram KA, Seake-Kwawu A, Weiss MG: Two-year evaluation of Intermittent Preventive Treatment for Children (IPTC) combined with timely home treatment for malaria control in Ghana. Malar J 2011, 10(1):127.

13. Ahorlu CK, Koram KA, Seakey AK, Weiss MG: Effectiveness of combined intermittent preventive treatment for children and timely home treatment for malaria control. Malar J 2009, 8:292.

14. Abdulla S, Schellenberg JA, Nathan R, Mukasa O, Marchant T, Smith T, Tanner M, Lengeler C: Impact on malaria morbidity of a programme supplying insecticide treated nets in children aged under 2 years in Tanzania: community cross sectional study. Bmj 2001, 322(7281):270-273.

15. Ayi I, Nonaka D, Adjovu JK, Hanafusa S, Jimba M, Bosompem KM, Mizoue T, Takeuchi T, Boakye DA, Kobayashi J: Research school-based participatory health education for malaria control in Ghana: engaging children as health messengers. Malar J 2010, 18:9-98.

16. Bojang KA, Akor F, Conteh L, Webb E, Bittaye O, Conway DJ, Jasseh M, Wiseman V, Milligan PJ, Greenwood B: Two strategies for the delivery of IPTc in an area of seasonal malaria transmission in The Gambia: a randomised controlled trial. PLoS Med 2011, 8(2):e1000409.

17. Castro MC, Tsuruta A, Kanamori S, Kannady K, Mkude S: Community-based environmental management for malaria control: evidence from a small-scale intervention in Dar es Salaam, Tanzania. Malar J 2009, 8(1):57

18. Eriksen J, Mujinja P, Warsame M, Nsimba S, Kouyate B, Gustafsson LL, Jahn A, Maller O, Sauerborn R, Tomson G: Effectiveness of a community intervention on malaria in rural Tanzania-a randomised controlled trial. Afr Health Sci 2010, 10(4):332-340.

19. D'Alessandro U, Langerock P, Bennett S, Francis N, Cham K, Greenwood BM: The impact of a national impregnated bed net programme on the outcome of pregnancy in primigravidae in The Gambia. Trans $R$ Soc Trop Med Hyg 1996, 90(5):487-492.

20. Dapeng $L$, Leyuan $S$, Xili $L$, Xiance $Y$ : A successful control programme for falciparum malaria in Xinyang, China. Trans R Soc Trop Med Hyg 1996, 90(2):100-102

21. Delacollette C, Stuyft PV, Molima K: Using community health workers for malaria control: experience in Zaire. Bull World Health Organ 1996, 74(4):423-430.

22. Ter Kuile FO, Terlouw DJ, Phillips-Howard PA, Hawley WA, Friedman JF, Kariuki SK, Shi YP, Kolczak MS, Lal AA, Vulule JM: Reduction of malaria during pregnancy by permethrin-treated bed nets in an area of intense perennial malaria transmission in western Kenya. Am J Trop Med Hyg 2003, 68(4 suppl):50-60.

23. Gies S, Coulibaly SO, Ouattara FT, Ky C, Brabin BJ, D'Alessandro U: A community effectiveness trial of strategies promoting intermittent preventive treatment with sulphadoxine-pyrimethamine in pregnant women in rural Burkina Faso. Malar J 2008, 7(1):180

24. Grabowsky M, Farrell N, Hawley W, Chimumbwa J, Hoyer S, Wolkon A, Selanikio J: Integrating insecticide-treated bednets into a measles vaccination campaign achieves high, rapid and equitable coverage with direct and voucher-based methods. Trop Med Int Health 2005, 10(11):1151-1160.

25. Grabowsky M, Nobiya T, Selanikio J: Sustained high coverage of insecticide-treated bednets through combined catch-up and keep-up strategies. Trop Med Int Health 2007, 12(7):815-822.

26. Greenwood BM, Greenwood AM, Snow RW, Byass P, Bennett S, Hatib-N'Jie $A B$ : The effects of malaria chemoprophylaxis given by traditional birth attendants on the course and outcome of pregnancy. Trans R Soc Trop Med Hyg 1989, 83(5):589-594.

27. Hightower A, Kiptui R, Manya A, Wolkon A, Eng JLV, Hamel M, Noor A, Sharif SK, Buluma R, Vulule J: Research bed net ownership in Kenya: the impact of 3.4 million free bed nets. Malar J 2010, 9:183.

28. Kidane G, Morrow RH: Teaching mothers to provide home treatment of malaria in Tigray, Ethiopia: a randomized trial. Lancet 2000, 356:550-555.

29. Kolaczinski JH, Kolaczinski K, Kyabayinze D, Strachan D, Temperley M, Wijayanandana N, Kilian A: Research costs and effects of two public sector delivery channels for long-lasting insecticidal nets in Uganda. Malar J 2010, 9:102.

30. Krezanoski PJ, Comfort AB, Hamer DH: Research effect of incentives on insecticide-treated bed net use in sub-Saharan Africa: a cluster randomized trial in Madagascar. Malar J 2010, 9:186.
31. Ter Kuile FO, Terlouw DJ, Kariuki SK, Phillips-Howard PA, Mirel LB, Hawley WA, Friedman JF, Shi YP, Kolczak MS, Lal AA: Impact of permethrin-treated bed nets on malaria, anemia, and growth in infants in an area of intense perennial malaria transmission in western Kenya. Am J Trop Med Hyg 2003, 68(4 suppl):68-77

32. Kweku M, Webster J, Adjuik M, Abudey S, Greenwood B, Chandramohan D: Options for the delivery of intermittent preventive treatment for malaria to children: a community randomised trial. PLoS One 2009, 4(9):e7256.

33. Macintyre K, Sosler S, Letipila F, Lochigan M, Hassig S, Omar SA, Githure J: A new tool for malaria prevention?: Results of a trial of permethrinimpregnated bedsheets (shukas) in an area of unstable transmission. Int J Epidemiol 2003, 32(1):157-160.

34. Mbonye AK, Bygbjerg IC, Magnussen P: Intermittent preventive treatment of malaria in pregnancy: a new delivery system and its effect on maternal health and pregnancy outcomes in Uganda. Bull World Health Organ 2008, 86:93-100.

35. Mbonye AK, Schultz Hansen K, Bygbjerg IC, Magnussen P: Effect of a community-based delivery of intermittent preventive treatment of malaria in pregnancy on treatment seeking for malaria at health units in Uganda. Public Health 2008, 122(5):516-525.

36. Msyamboza KP, Savage EJ, Kazembe PN, Gies S, Kalanda G, D'Alessandro U, Brabin BJ: Community-based distribution of sulfadoxine-pyrimethamine for intermittent preventive treatment of malaria during pregnancy improved coverage but reduced antenatal attendance in southern Malawi. Trop Med Int Health 2009, 14(2):183-189.

37. Noor AM, Amin AA, Akhwale WS, Snow RW: Increasing coverage and decreasing inequity in insecticide-treated bed net use among rural Kenyan children. PLoS Med 2007, 4(8):e255.

38. Okabayashi $H$, Thongthien $P$, Singhasvanon P, Waikagul J, Looareesuwan $S$, Jimba M, Kano S, Kojima S, Takeuchi T, Kobayashi J, Tateno S: Keys to success for a school-based malaria control program in primary schools in Thailand. Parasitology international 2006, 55(2):121-126.

39. Okeibunor JC, Orji BC, Brieger W, Ishola G, Otolorin ED, Rawlins B, Ndekhedehe EU, Onyeneho N, Fink G: Preventing malaria in pregnancy through community-directed interventions: evidence from Akwa Ibom State, Nigeria. Malar J 2011, 10(1):227

40. Rhee M, Sissoko M, Perry S, McFarland W, Parsonnet J, Doumbo O: Use of insecticide-treated nets (ITNs) following a malaria education intervention in Piron, Mali: a control trial with systematic allocation of households. Malar J 2005, 4(1):35.

41. Schellenberg D, Menendez C, Kahigwa E, Aponte J, Vidal J, Tanner M, Mshinda $\mathrm{H}$, Alonso P: Intermittent treatment for malaria and anaemia control at time of routine vaccinations in Tanzanian infants: a randomised, placebo-controlled trial. Lancet 2001, 357(9267):1471-1477.

42. Sharma SK, Tyagi PK, Upadhyay AK, Haque MA, Mohanty SS, Raghavendra K, Dash AP: Efficacy of permethrin treated long-lasting insecticidal nets on malaria transmission and observations on the perceived side effects, collateral benefits and human safety in a hyperendemic tribal area of Orissa, India. Acta Tropica 2009, 112(2):181-187.

43. Skarbinski J, Massaga JJ, Rowe AK, Kachur SP: Distribution of free untreated bednets bundled with insecticide via an integrated child health campaign in Lindi Region, Tanzania: lessons for future campaigns. Am J Trop Med Hyg 2007, 76(6):1100-1106.

44. Staedke SG, Mwebaza N, Kamya MR, Clark TD, Dorsey G, Rosenthal PJ, Whitty CJM: Home management of malaria with artemether-lumefantrine compared with standard care in urban Ugandan children: a randomised controlled trial. Lancet 2009, 373(9675):1623-1631.

45. Tagbor H, Cairns M, Nakwa E, Browne E, Sarkodie B, Counihan H, Meek S, Chandramohan D: The clinical impact of combining intermittent preventive treatment with home management of malaria in children aged below 5 years: cluster randomised trial. Trop Med Int Health 2011, 16(3):280-289.

46. Terlouw DJ, Morgah K, Wolkon A, Dare A, Dorkenoo A, Eliades MJ, Eng JV, Sodahlon YK, ter Kuile FO, Hawley WA: Impact of mass distribution of free long-lasting insecticidal nets on childhood malaria morbidity: the Togo national integrated child health campaign. Malar J 2010, 9:199.

47. Thang ND, Erhart A, Hung LX, Thuan LK, Xa NX, Thanh NN, Ky PV, Coosemans M, Speybroeck N, D'Alessandro U: Rapid descrese of malaria morbidity following the introduction of community-based monitoring in a rural area of central Vietnam. Malar J 2009, 8(3). doi:10.1186/ 1475-2875-1188-1183. 
48. Thwing J, Hochberg N, Eng JV, Issifi S, James Eliades M, Minkoulou E, Wolkon A, Gado H, Ibrahim O, Newman RD: Insecticide-treated net ownership and usage in Niger after a nationwide integrated campaign. Trop Med Int Health 2008, 13(6):827-834.

49. Wolkon A, Eng JLV, Morgah K, Eliades MJ, Thwing J, Terlouw DJ, Takpa V, Dare A, Sodahlon YK, Doumanou Y: Rapid scale-up of long-lasting insecticide-treated bed nets through integration into the national immunization program during child health week in Togo, 2004. Am J Trop Med Hyg 2010, 83(5):1014-1019.

50. D'Alessandro U, Olaleye B, Langerock P, Aikins MK, Thomson MC, Cham MK, Greenwood BM, McGuire W, Bennett S, Cham BA: Mortality and morbidity from malaria in Gambian children after introduction of an impregnated bednet programme. Lancet 1995, 345(8948):479-483.

51. Singh M, Brown G, Rogerson SJ: Ownership and use of insecticide-treated nets during pregnancy in sub-Saharan Africa: a review. Challenge 2013, 1:4

52. Hill J, Hoyt J, van Eijk AM, D'Mello-Guyett L, ter Kuile FO, Steketee R, Smith H, Webster J: Factors affecting the delivery, access, and use of interventions to prevent malaria in pregnancy in sub-Saharan Africa: a systematic review and meta-analysis. PLoS Med 2013, 10(7):e1001488.

53. Malm KL, Segbaya S, Forson I, Gakpey KD, Sampong LB, Adjei EA, Bart-Plange C: Implementation of the Affordable Medicines for Malaria Facility (AMFm) in Ghana: processes, challenges and achievements. J Public Health Policy 2013, 34(2):302-314.

54. Kizito J, Kayendeke M, Nabirye C, Staedke SG, Chandler CIR: Improving access to health care for malaria in Africa: a review of literature on what attracts patients. Malar J 2012, 11(1):55.

55. Kinung'hi SM, Mashauri F, Mwanga JR, Nnko SE, Kaatano GM, Malima R, Kishamawe C, Magesa S, Mboera LEG: Knowledge, attitudes and practices about malaria among communities: comparing epidemic and non-epidemic prone communities of Muleba district, North-western Tanzania. BMC Public Health 2010, 10(1):395.

56. USAIDS: Decades of Progress: USAID's Child Survival and Maternal Health Program; 2009. Available at http://pdf.usaid.gov/pdf_docs/PDACN044.pdf

57. Kalyango JN, Rutebemberwa E, Alfven T, Ssali S, Peterson S, Karamagi C: Performance of community health workers under integrated community case management of childhood illnesses in eastern Uganda. Malar J 2012, 11:282.

58. Cardemil CV, Gilroy KE, Callaghan-Koru JA, Nsona H, Bryce J: Comparison of methods for assessing quality of care for community case management of sick children: an application with community health workers in Malawi. Am J Trop Med Hyg 2012, 87(5 Suppl):127-136.

59. UNICEF: Child Health Days 1999-2009: Key Achievements and the Way Forward. In A Report Prepared for the UNICEF Joint Working Group on Child Health Days. New York: UNICEF; 2011.

60. Oliphant NP, Mason JB, Doherty T, Chopra M, Mann P, Tomlinson M, Nsibande D, Mebrahtu S: The contribution of child health days to improving coverage of periodic interventions in six African countries. Food Nutr Bull 2010, 31(Supplement 3):248S-263S.

61. Collins DH, Jarrah Z, Wright KD, Lee AC: The cost of integrated community health services for treating child pneumonia, diarrhoea, and malaria in three African countries: economic research using systematic sampling. Lancet 2013, 381:S31.

62. Kern E, Verguet S, Yuhas K, Odhiambo FH, Kahn JG, Walson J: Provision of bednets and water filters to delay HIV-1 progression: cost-effectiveness analysis of a Kenyan multisite study. Trop Med Int Health 2013, 18(8):916-924.

doi:10.1186/2049-9957-3-25

Cite this article as: Salam et al:: Impact of community-based interventions for the prevention and control of malaria on intervention coverage and health outcomes for the prevention and control of malaria. Infectious Diseases of Poverty 2014 3:25.

\section{Submit your next manuscript to BioMed Central and take full advantage of:}

- Convenient online submission

- Thorough peer review

- No space constraints or color figure charges

- Immediate publication on acceptance

- Inclusion in PubMed, CAS, Scopus and Google Scholar

- Research which is freely available for redistribution

Submit your manuscript at www.biomedcentral.com/submit
C Biomed Central 